\title{
A Simple Linkage for Describing Equal Areas.
}

\author{
By E. M. Horsburgh.
}

(Read 13th June 1913. Received Q20nd June 1913).

If $\mathrm{H}$ (Fig. 1) be the middle point of a straight bar QP and if a straight bar $\mathrm{OH}$ of length one-half of $\mathrm{QP}$ be pin-jointed to $\mathrm{QP}$ at $H$, a simple linkage is formed, which may be called a $T$-linkage.

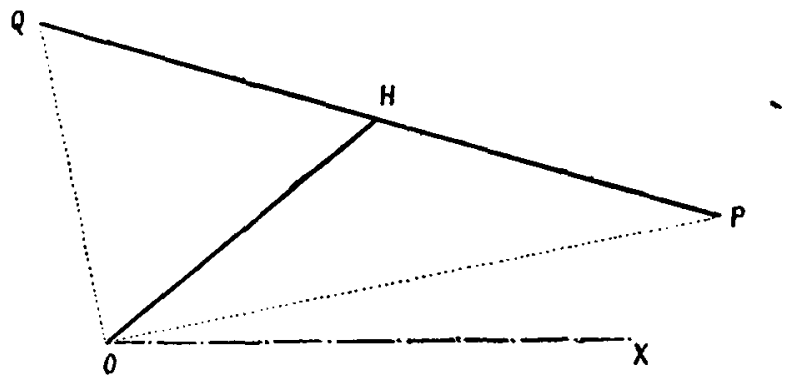

Fig. 1.

This, with an additional fixed bar guiding $\mathrm{P}$ or $\mathrm{Q}$ in a straight line through $O$, forms a well-known ellipsograph, or an equally well-known parallel motion. Let the $\mathrm{T}$-linkage be placed in the plane of the paper and let $O$ be taken as pole, so that the linkage is free to turn about $O$ in the plane of the paper. Then if $P$ describe any closed curve not enclosing $O, Q$ will describe a corresponding curve of equal area: For if the coordinates of $\mathrm{P}$ and $\mathrm{Q}$ be $(r, \theta)$ and $(\rho, \alpha)$ respectively, and if $a$ ibe the length of $\mathrm{OH}$, the equations of the transformation are

$$
\text { (i) } r^{2}+\rho^{2}=4 a^{2}, \quad \text { (ii) } a=\frac{\pi}{2}+0 \text {. }
$$

Accordingly if $\mathrm{P}$ describe the curve $f(r, \theta)=0, \mathrm{Q}$ will describe the curve $f\left(\sqrt{4} a^{2}-\rho^{2}, \alpha-\frac{\pi}{2}\right)=0$. Multiplying (i) by $1_{2}^{1} d \theta$ and integrating on the supposition that $P$ describes a closed curve such that $P Q$ returns to its original position without making a circuit about $O$, then $\frac{1}{2} \int r^{2} d \theta+\frac{1}{2} \int \rho^{2} \delta \alpha=0$ taken round the curve, so that 
if $A_{1}$ and $A_{2}$ be the areas described by $P$ and $Q$ respectively, $A_{1}=-A_{2}$.

The equations of the transformed curves are most frequently complicated, but sometimes simple degenerate cases occur. Thus the family of straight lines $\theta=c$ transforms into the family of straight lines $\alpha=\frac{\pi}{2}+c$, and the family of concentric circles $r=k$ into the family of concentric circles $\rho=\sqrt{4 a^{2}-k^{2}}$, and thus the orthogonal network composed of the lines $\theta=c$ and the circles $r=k$ transforms into an equal area orthogonal network. (Figs. 2 and 3 ).

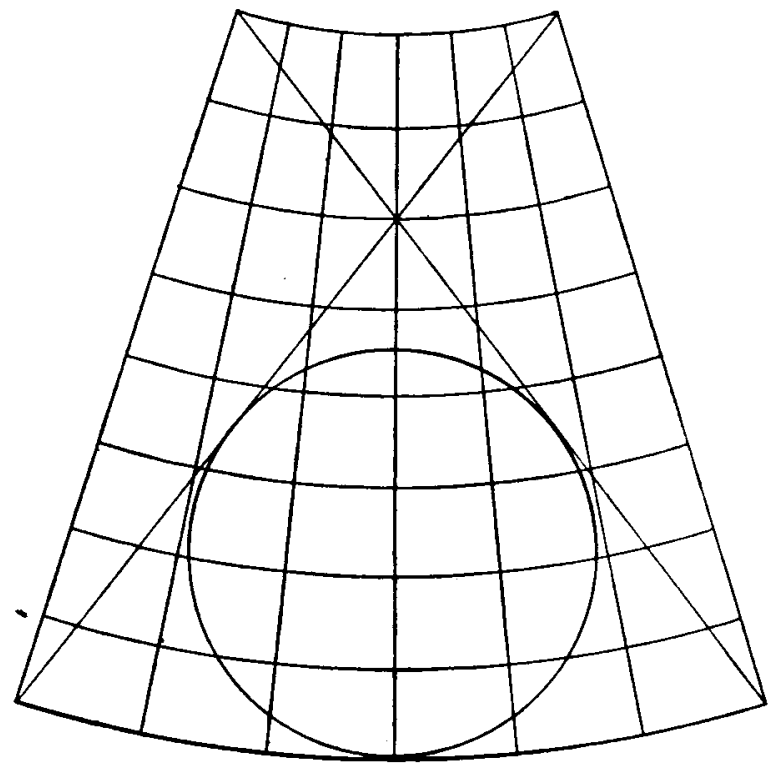

Fig. 2.

This suggested an application to some of the equal-area worldmaps since the linkage deduces from any figure an infinite number of others all of equal area. Figs. 4, 5, 6, are representations of well-known equal-area world-maps, the first two by Lambert, the last by Collignon. Figs. 7, 8, 9, show equal-area maps deduced by the linkage and corrected with regard to the reversed 
orientation which arises from the fact that $\mathrm{P}$ and $\mathrm{Q}$ describe figures in opposite senses.

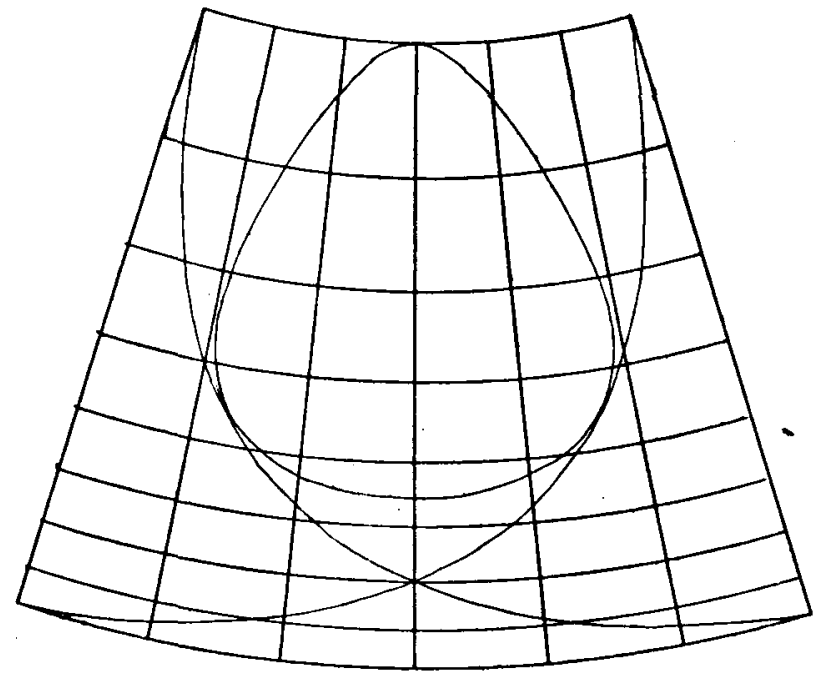

Fig. 3.

If the points $\mathrm{P}$ and $\mathrm{Q}$ describe arcs of curves instead of closed contours, then

$$
\frac{1}{2} \int_{\theta_{1}}^{\theta_{2}} r^{2} d \theta+\frac{1}{2} \int_{a_{1}}^{a_{2}} \rho^{2} \delta \alpha=2 a^{2}\left(\theta_{2}-\theta_{1}\right)
$$

where $\theta_{1}, \alpha_{1}$ correspond to the initial, and $\theta_{2}, \alpha_{2}$ to the final positions of the tracing points. Thus the sum of the areas of the two sectors described by $\mathbf{P}$ and $\mathbf{Q}$ about the origin is equal to the area of the sector of a circle of radius $P Q$ which contains the angle $\theta_{2}-\theta_{1}$. If the linkage make a complete revolution about the pole $O, P Q$ turns through an angle $2 \pi$, and the sum of the areas of the sectors traced by $P$ and $Q$ is equal to the area of a circle of radius $P Q$.

When one tracing point describes a curve passing through the pole an indeterminate condition arises, and a contour described twice by one tracing point may correspond to one described once by the other.

By taking a tracing point at any position $S$ in $P Q$, an area can be drawn which is any required fraction of a given area $A$. If $\mathrm{QS}=c$ and $\mathrm{SP}=c^{\prime}$, we have by a particular case of Holditch's Theorem that the area described by $S$ is $A\left(c-c^{\prime}\right) /\left(c+c^{\prime}\right)$ when $P Q$ rocks back to its original position. 

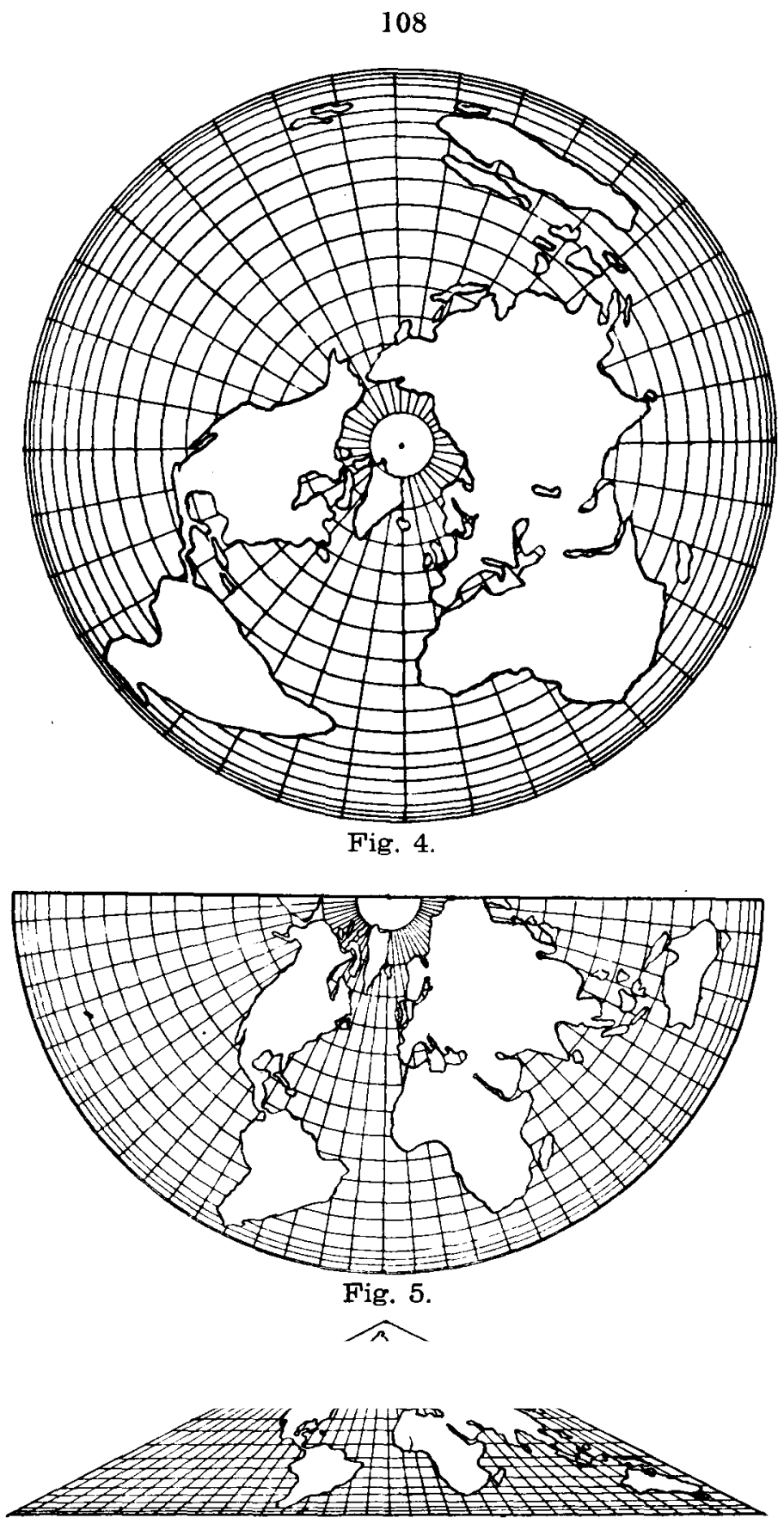


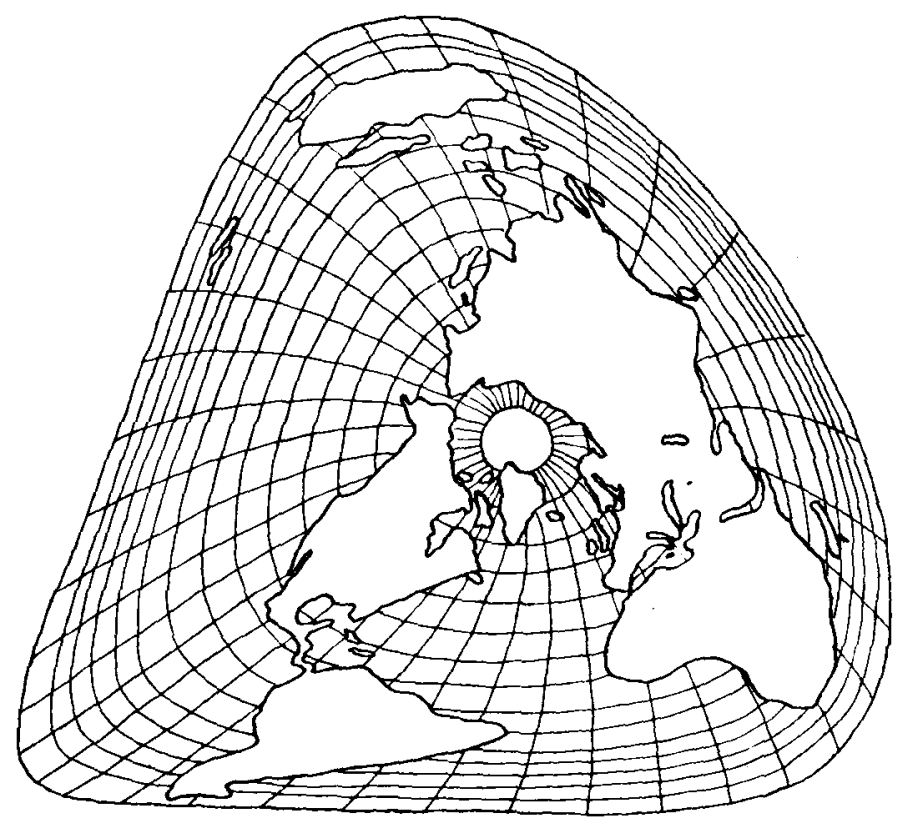

Fig. 7.

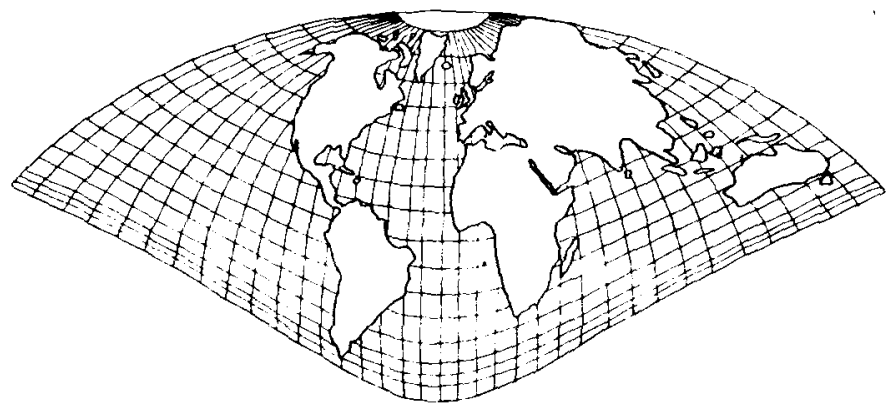

Fig. 8.

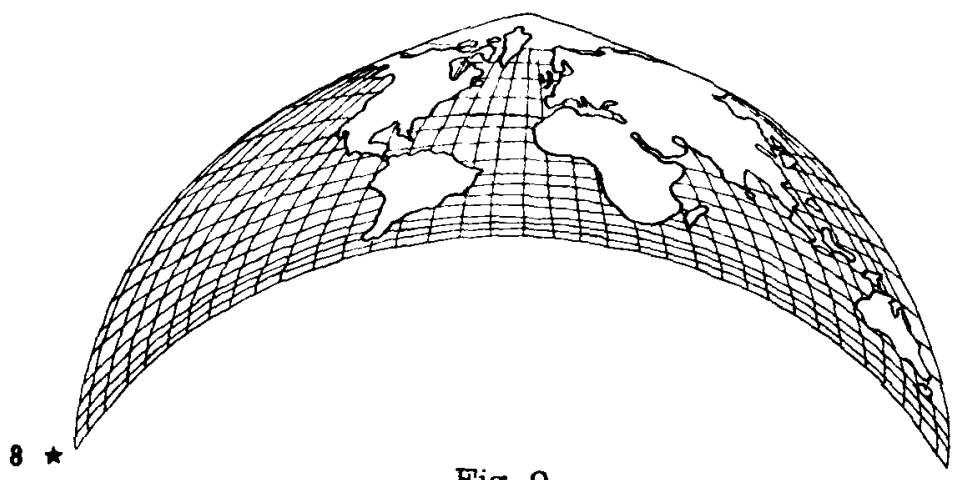

Fig. 9. 
If $(r, \theta)$ and $(\rho, a)$ be corresponding points on a curve and its transformed curve, and $\phi$ and $\Phi$ the angles between the radius vector and the tangent, then

$$
\tan \phi=\frac{r d \theta}{d r}, \quad \tan \Phi=\frac{\rho d \alpha}{d \rho} .
$$

Hence, since $r d r+\rho d \rho=0$ and $d \alpha=d \theta$

$$
\frac{\tan \phi}{\tan \Phi}=-\frac{r^{2}}{\rho^{2}} \text {. }
$$

If $\phi^{\prime}$ and $\Phi^{\prime}$ be the angles for another corresponding pair of curves through these points, then

$$
\frac{\tan \phi^{\prime}}{\tan \Phi^{\prime}}=-\frac{r^{2}}{\rho^{2}}=\frac{\tan \phi}{\tan \Phi}
$$

which gives the law of intersection for the transformed network. 